\title{
Socialización de estudiantes universitarios en el contexto de la educación a distancia
}

\section{Socialization of university students in the context of distance learning}

\author{
Marina V. Bryantseva \\ mv.bryantseva@mgou.ru \\ Moscow Region State University, Moscow, Russia. \\ https://orcid.org/0000-0002-4751-5883 \\ Ekaterina I. Artamonova \\ ei.artamonova@mgou.ru \\ Moscow Region State University, Mytishchi, Russia. \\ https://orcid.org/0000-0002-9181-8005 \\ Olga V. Dybina \\ dybinaov@yandex.ru \\ Togliatti State University, Togliatti, Russia. \\ https://orcid.org/0000-0002-0146-3336 \\ Anna I. Orlova \\ annasamurina@mail.ru \\ Nizhny Novgorod State Engineering and Economic University, Nizhny Novgorod, Russia. \\ https://orcid.org/0000-0001-8708-6537 \\ Irina A. Kuznetsova \\ nosovair@rambler.ru \\ Nizhny Novgorod State Engineering and Economic University, Nizhny Novgorod, Russia. \\ https://orcid.org/0000-0001-7086-0290
}

Recibido: $18 / 12 / 2020$

Aceptado: $14 / 01 / 2021$

\section{Resumen}

El propósito del artículo fue analizar la socialización de los estudiantes en el proceso de aprendizaje a distancia. La metodología del estudio involucró a estudiantes de instituciones de educación superior. Para realizar la investigación, utilizamos métodos para determinar las cualidades de liderazgo de los estudiantes en el proceso de interacción educativa. Se destacan los criterios por los cuales se determinó el nivel de socialización de los participantes de la investigación. Como conclusión en el contexto de la transición a la educación a distancia, los estudiantes de primer año experimentan las mayores dificultades. A partir de los datos obtenidos, podemos hablar de la necesidad de seguir mejorando el proceso de socialización y tomar medidas 
Marina V. Bryantseva, Ekaterina I. Artamonova, Olga V. Dybina, Anna I. Orlova, Irina A. Kuznetsova.

adicionales para adaptar a los estudiantes de primer año al proceso educativo en la universidad.

Palabras clave: socialización, adaptación, educación profesional, educación a distancia, proceso educativo, cualidades de liderazgo.

\section{Abstract}

The purpose of the article was to analyze the socialization of students in the distance learning process. The study methodology involved students from higher education institutions. To conduct the research, we used methods to determine the leadership qualities of students in the process of educational interaction. The criteria by which the level of socialization of the research participants was determined are highlighted. As a conclusion, in the context of the transition to distance education, first-year students experience the greatest difficulties. Based on the data obtained, we can talk about the need to continue improving the socialization process and take additional measures to adapt first-year students to the educational process at the university.

Key Words: socialization, adaptation, professional education, distance learning, educational process, leadership qualities.

\section{Introducción}

Los requisitos de la legislación moderna incluyen la implementación de tecnologías innovadoras de educación a distancia por parte de instituciones de educación superior. En el contexto de una transición completa al aprendizaje a distancia, muchas dificultades afectan varios aspectos de la vida de los estudiantes. En primer lugar, el aprendizaje a distancia tiene un fuerte impacto en la socialización de los estudiantes, que ocupa uno de los lugares más importantes en el proceso de su formación y la formación de la competencia profesional. El estudio tiene como objetivo analizar la socialización de los estudiantes en el proceso de aprendizaje a distancia.

La interacción remota de sujetos del proceso educativo en las instituciones de educación superior se lleva a cabo con unas herramientas electrónicas que promueven la interacción virtual y la organización del proceso educativo (Shcerbakova \& Shcerbakova, 2019). Implementadas durante mucho tiempo en un entorno de aprendizaje mixto, las herramientas son familiares para los estudiantes de pregrado (Vaganova et al., 2020a). El alumno actúa como sujeto capaz de planificar, organizar y dirigir sus actividades en el proceso de aprendizaje. Su socialización se refleja en la posesión de habilidades de comunicación constructiva, la capacidad de prevenir conflictos y su resolución independiente. Las cualidades de liderazgo muestran la capacidad de los estudiantes para organizar sus actividades y las actividades del equipo para lograr sus objetivos educativos (Kidina, 2020). 
Una de las etapas iniciales de socialización es la adaptación - adaptación de los estudiantes a la naturaleza, contenido y condiciones del proceso de aprendizaje (Vaganova et al, 2019a). su período se caracteriza por la habitación del estudiante al desarrollo de una gran cantidad de información, la duración y formas de las clases, nuevos requisitos. El alumno desarrolla la capacidad de estudiar de forma independiente el material, distribuir su tiempo y esfuerzo para completar las tareas. La interacción activa con los compañeros facilita que el alumno pase por el proceso de adaptación, ya que forma parte de un solo equipo en el que los alumnos se apoyan entre sí, resolviendo conjuntamente cuestiones emergentes. En el contexto del aprendizaje a distancia, los estudiantes no tienen la oportunidad de una comunicación real y un apoyo real. Los primeros cursos dominan de forma independiente las nuevas capacidades técnicas para ellos. La comunicación con los compañeros se reduce al mínimo. Como resultado, el proceso de adaptación al proceso educativo y de socialización es más largo. Crear las condiciones para una socialización exitosa es una de las prioridades de las instituciones de educación superior.

\section{Marco teórico y revision de literatura}

La socialización fue considerada por científicos desde diferentes posiciones. Muchos investigadores consideran que la función principal de la socialización es la familiarización con el entorno social, la conciencia colectiva (Pichugina \& Bondarchuk, 2019). Observando que una persona al mismo tiempo suprime sus intereses para unirse a la sociedad. Sin embargo, existe una opinión contraria. Los científicos que se adhieren a la segunda posición designan la función principal de la socialización como la formación de una personalidad única. En el proceso de ingresar al espacio social, una persona se familiariza con nuevos valores y roles, domina la experiencia social, se vuelve más independiente, capaz de evaluación y autoevaluación y adquiere autonomía (Toktarova \& Fedorova, 2019).

La socialización de la personalidad se considera en las obras de V. A. Lukov, E. E. Lukova y otros. La socialización se ve con mayor frecuencia como un proceso bidireccional (Lukov y Lukova, 2014). En el primer caso, se trata de la transferencia de valores culturales por parte de la sociedad a cada individuo; en el segundo, la percepción de los valores culturales de la sociedad por parte de una persona, su desarrollo a lo largo de la vida. La socialización tiene lugar solo bajo la condición de interacción continua con la realidad.

El desarrollo de la socialización se da a través de actividades conjuntas, interacción y participación activa en actividades prácticas. Así mismo, la socialización implica no solo la asimilación de la experiencia de un individuo, sino también su transformación (Vaganova et al., 2020b). Consideramos la socialización como parte del proceso de aprendizaje a distancia en la universidad (Dobudko et al., 2019b). 
Los principios de la socialización son: actividad, coherencia, implicación personal y actividad. El principio de actividad asume la interacción activa de los estudiantes en el proceso de resolución de diversos problemas, continuidad e integridad del proceso de asimilación de la realidad objetiva por parte de los estudiantes (Dobudko et al., 2019a). El principio de coherencia implica el impacto en la personalidad del alumno y el entorno externo e interno. El principio de inclusión personal nos permite presentar a la persona como sujeto activo del proceso educativo.

Los profesores, su competencia, inteligencia y estilo de pensamiento juegan un papel importante en la socialización. Un profesor competente puede construir un proceso de socialización competente teniendo en cuenta las características psicológicas, la individualización de la formación y otros factores. Sin embargo, en condiciones remotas, sin interacción directa, estos procesos son difíciles. Por lo tanto, el docente utiliza tecnologías electrónicas modernas con la ayuda de las cuales activa la independencia de los estudiantes, actuando como consultor.

La socialización profesional se entiende como el proceso de entrada de un individuo al entorno profesional. En el proceso de socialización profesional, el alumno domina la experiencia profesional, los tipos de conducta en la comunidad, desarrolla decisiones de conducta adecuadas que implican un continuo desarrollo profesional y autodesarrollo (Klimenko, 2012).

En el proceso de formación se deben formar competencias profesionales, desarrollar cualidades personales profesionales y fundamentos ideológicos (Tyumaseva et al., 2018). Sin embargo, en el contexto de una transición completa al aprendizaje en línea, existen muchas dificultades asociadas con varios aspectos de la socialización y adaptación de los estudiantes (Ponachugin, 2020). La adaptación de los estudiantes al aprendizaje en un entorno electrónico se considera en el trabajo de V. I. Toktarova, S. N. Fedorova (Toktarova \& Fedorova, 2019). La atención de los investigadores al proceso de adaptación de los estudiantes está condicionada por las necesidades objetivas de la sociedad moderna de especialistas calificados capaces de resolver problemas en interacción (Eliseeva et al., 2020).

\section{Metodología}

El estudio tiene como objetivo estudiar el nivel de socialización de los estudiantes en las condiciones de la educación a distancia en las escuelas superiores. Los participantes del estudio fueron estudiantes de instituciones de educación superior en el número de 324 personas del primero al cuarto año. La edad de los encuestados osciló entre los 18 y los 24 años. La adaptación es una parte importante de la socialización de los estudiantes. La capacidad de los estudiantes para adaptarse al entorno educativo profesional se evaluó mediante los siguientes indicadores: habilidades comunicativas y organizativas, enfoque creativo de las tareas, capacidad para establecer relaciones con los sujetos educativos. La socialización se refleja en la capacidad del alumno para mostrar cualidades de liderazgo, independencia y 
responsabilidad por sus propias decisiones y las decisiones de los grupos de los que es miembro, en el proceso de consecución de sus objetivos (Nagovitsyn et al., 2020).

En el curso del estudio se utilizó el método de Autoevaluación de las cualidades de liderazgo, según el cual los estudiantes deben estar de acuerdo con las declaraciones propuestas o refutarlas. 4 puntos significan "de acuerdo", 3 puntos - "de acuerdo en lugar de en desacuerdo", 2 puntos - "difícil de decir", 1 - "en desacuerdo en lugar de estar de acuerdo", 0 - "completamente en desacuerdo". Un fragmento del cuestionario se presenta en la Tabla 1.

Tabla 1. Cuestionario de autoevaluación de liderazgo

\begin{tabular}{lc}
\hline \multicolumn{1}{c}{ Pregunta } & $\begin{array}{c}\text { ¿Estás de acuerdo con esta afirmación? } \\
(4-\text { totalmente de acuerdo, } 0 \text { - totalmente } \\
\text { en desacuerdo) }\end{array}$ \\
\hline $\begin{array}{l}\text { Yo puedo superar las dificultades y sé cómo } \\
\text { hacerlo }\end{array}$ & $0-4$ \\
\hline $\begin{array}{l}\text { Sé involucrar a mis compañeros en una causa } \\
\text { común }\end{array}$ & $0-4$ \\
\hline Yo puedo convencer a mis compañeros de clase & $0-4$ \\
\hline Planeo mi tiempo y trabajo bien & $0-4$ \\
\hline Normalmente logro lo que me propongo & $0-4$ \\
\hline $\begin{array}{l}\text { En las relaciones con las personas, logro el } \\
\text { entendimiento mutuo. }\end{array}$ & $0-4$ \\
\hline $\begin{array}{l}\text { Utilizo las experiencias de otras personas para } \\
\text { resolver problemas. }\end{array}$ & $0-4$ \\
\hline $\begin{array}{l}\text { Puedo controlar mi trabajo y el trabajo de mi } \\
\text { equipo. }\end{array}$ & $0-4$ \\
\hline
\end{tabular}

La cantidad total de puntos determina el desarrollo de las siguientes cualidades de liderazgo que muestra un alumno en el proceso de aprendizaje a distancia: la capacidad de autogestionarse, el conocimiento de los objetivos, la capacidad de resolver problemas y salir de situaciones difíciles, las habilidades organizativas, la capacidad para trabajar en grupo.

El estudio también utilizó el método de "Efectividad del liderazgo" de RS Nemov, que asume que la respuesta es "sí" o "no" (Nemov, 1999). La encuesta ayuda a determinar si un alumno es capaz de ser líder en las actividades prácticas y en el futuro ámbito profesional. Un fragmento del cuestionario se presenta en la Tabla 2. 
Marina V. Bryantseva, Ekaterina I. Artamonova, Olga V. Dybina, Anna I. Orlova, Irina A. Kuznetsova.

Tabla 2. Cuestionario de eficacia del liderazgo

\begin{tabular}{lc}
\hline \multicolumn{1}{c}{ Pregunta } & Responder \\
\hline ¿Te esfuerzas por colaborar con otros sujetos del proceso educativo? & $(\mathrm{si} / \mathrm{no})$ \\
\hline ¿Con qué frecuencia demuestras creatividad al completar las tareas? & $(\mathrm{si} / \mathrm{no})$ \\
\hline ¿Eres lo suficientemente flexible a la hora de tomar decisiones? & $(\mathrm{si} / \mathrm{no})$ \\
\hline ¿Explica a los demás las razones de esta o aquella decisión? & $(\mathrm{si} / \mathrm{no})$ \\
\hline ¿Tus compañeros confían en ti? & $(\mathrm{si} / \mathrm{no})$ \\
\hline ¿Sabes cómo ser un oyente atento? & $(\mathrm{si} / \mathrm{no})$ \\
\hline ¿Te gusta hacer un trabajo desafiante pero interesante? & $(\mathrm{si} / \mathrm{no})$ \\
\hline
\end{tabular}

Por cada respuesta positiva, el sujeto recibe 1 punto, por cada respuesta negativa - 0 puntos. Si el sujeto obtiene una puntuación de 30 a 40 puntos, entonces su posición de liderazgo en la realización de tareas colectivas en el aprendizaje a distancia se reconoce como altamente eficaz. Si la cantidad de puntos anotados está en el rango de 11 a 29, el promedio efectivo. Si la cantidad era 10 o menos, entonces la posición de liderazgo se evalúa como ineficaz.

La evaluación de la efectividad de la socialización de los estudiantes se realizó de acuerdo con los siguientes criterios: motivacional, cognitivo, comunicativo, creativoactividad, emocional-volitivo.

Las características de los criterios se presentan en la tabla 3.

Tabla 3. Características de los criterios de socialización de los estudiantes en el contexto de la educación a distancia

\begin{tabular}{lll}
\hline No. & \multicolumn{1}{c}{ Criterio } & \multicolumn{1}{c}{ Característica } \\
\hline $\mathbf{1}$ & Motivacional & $\begin{array}{l}\text { Demuestra compromiso con el alumno y la implementación de una } \\
\text { actividad profesional independiente; Manifestar tolerancia y soluciones } \\
\text { de forma racional y directa, alto nivel de responsabilidad. }\end{array}$ \\
\hline $\mathbf{2}$ & Cognitivo & $\begin{array}{l}\text { Refleja el interés del estudiante en futuras actividades profesionales, } \\
\text { construyendo relaciones en el proceso de la actividad cognitiva; } \\
\text { determinación, ingenio, toma de decisiones rápida }\end{array}$ \\
\hline $\mathbf{3}$ & Comunicativo & $\begin{array}{l}\text { La capacidad del estudiante para entablar relaciones con otras materias } \\
\text { del proceso educativo, comportamiento en situaciones difíciles, elección } \\
\text { del estilo de comportamiento. }\end{array}$ \\
\hline $\mathbf{4}$ & Actividad creativa & $\begin{array}{l}\text { Refleja la capacidad del alumno para mostrar su componente creativo, } \\
\text { utilizar las herramientas necesarias para la interacción, organizar el } \\
\text { proceso para lograr metas conjuntas, distribuir funciones en subgrupos, } \\
\text { tomar decisiones atípicas }\end{array}$ \\
\hline $\mathbf{5}$ & $\begin{array}{l}\text { Emocionalmente } \\
\text { voluntad fuerte }\end{array}$ & $\begin{array}{l}\text { Refleja estabilidad moral, optimismo, sentido del tacto, enfoque en el } \\
\text { éxito, la capacidad de percibir críticas, intentos fallidos de lograr } \\
\text { resultados y sacar conclusiones, la capacidad de caracterizar a otras } \\
\text { personas, para notar sus lados positivos y negativos. }\end{array}$ \\
& &
\end{tabular}

Los criterios presentados determinarán el nivel de socialización de los estudiantes de las instituciones de educación superior. 


\section{Resultados y discusión}

El sistema de educación a distancia en las universidades estudiadas es un amplio abanico de diversas herramientas y tecnologías electrónicas utilizadas por los sujetos del proceso educativo. Las clases regulares se llevan a cabo utilizando la plataforma electrónica Zoom. La plataforma le permite recrear condiciones cercanas al proceso de aprendizaje a tiempo completo. Cada participante del seminario web en línea puede hablar y hacer las preguntas necesarias. Las presentaciones, los proyectos de protección y la puesta en escena de los juegos tienen lugar en el espacio virtual.

Las tecnologías electrónicas innovadoras introducidas en la práctica de las universidades se han implementado con éxito durante varios años (Nikonova et al., 2019a). El uso de medios técnicos para la interacción de aprendizaje remoto es común para los estudiantes de último año. En un entorno de aprendizaje mixto, realizaban tareas grupales con regularidad utilizando Moodle, Skype y otras herramientas electrónicas. Con la transición completa al aprendizaje a distancia, su comunicación y socialización adquirieron características específicas. Sin embargo, ellos, como estudiantes con la experiencia de la interacción virtual, continuaron sus estudios con las menores dificultades, a diferencia de los estudiantes de primer año.

El proceso de socialización de los cursos junior tiene una característica específica, que consiste principalmente en la baja adaptación de los estudiantes al proceso educativo en el conjunto de la universidad. Las dificultades de los estudiantes se deben a la falta de interacción y comunicación real con los compañeros, así como al aspecto técnico del tema.

Utilizando los métodos de RS Nemov, pudimos determinar el número de estudiantes con cualidades de liderazgo que reflejan la socialización y son necesarios para la implementación de futuras actividades profesionales.

Los resultados de la aplicación del método "Autoevaluación de las cualidades de liderazgo" entre 1-4 cursos mostraron que se observa un bajo nivel de autoevaluación de las cualidades de liderazgo en los estudiantes de primer año.

Tabla 4. Resultados de la aplicación de la metodología "Autoevaluación de las cualidades de liderazgo"

\begin{tabular}{llll}
\hline Curso & Nivel alto & Nivel medio & Nivel bajo \\
\hline $\mathbf{1}$ & $26 \%$ & $30 \%$ & $44 \%$ \\
\hline $\mathbf{2}$ & $28 \%$ & $32 \%$ & $40 \%$ \\
\hline $\mathbf{3}$ & $29 \%$ & $41 \%$ & $30 \%$ \\
\hline $\mathbf{4}$ & $32 \%$ & $45 \%$ & $23 \%$ \\
\hline
\end{tabular}


Los resultados de la autoevaluación muestran que los estudiantes de primer año valoran bastante sus cualidades de liderazgo. Sin embargo, en condiciones remotas, como señalan los propios estudiantes, puede resultarles difícil establecer relaciones en subgrupos cuando realizan tareas. Los resultados de los cursos superiores son ligeramente superiores.

Tabla 5. Resultados de la Metodología de Efectividad del Liderazgo

\begin{tabular}{llll}
\hline Curso & Nivel alto & Nivel medio & Nivel bajo \\
\hline $\mathbf{1}$ & $15 \%$ & $35 \%$ & cincuenta\% \\
\hline $\mathbf{2}$ & $17 \%$ & $36 \%$ & $47 \%$ \\
\hline $\mathbf{3}$ & $35 \%$ & $38 \%$ & $27 \%$ \\
\hline $\mathbf{4}$ & $38 \%$ & $40 \%$ & $22 \%$ \\
\hline
\end{tabular}

El porcentaje de estudiantes con un bajo nivel de liderazgo disminuye al 4ํaño. Los estudiantes adquieren las cualidades necesarias para interactuar en equipo y lograr los resultados planificados. En el primer año, solo el 15\% de los estudiantes tiene un nivel alto.

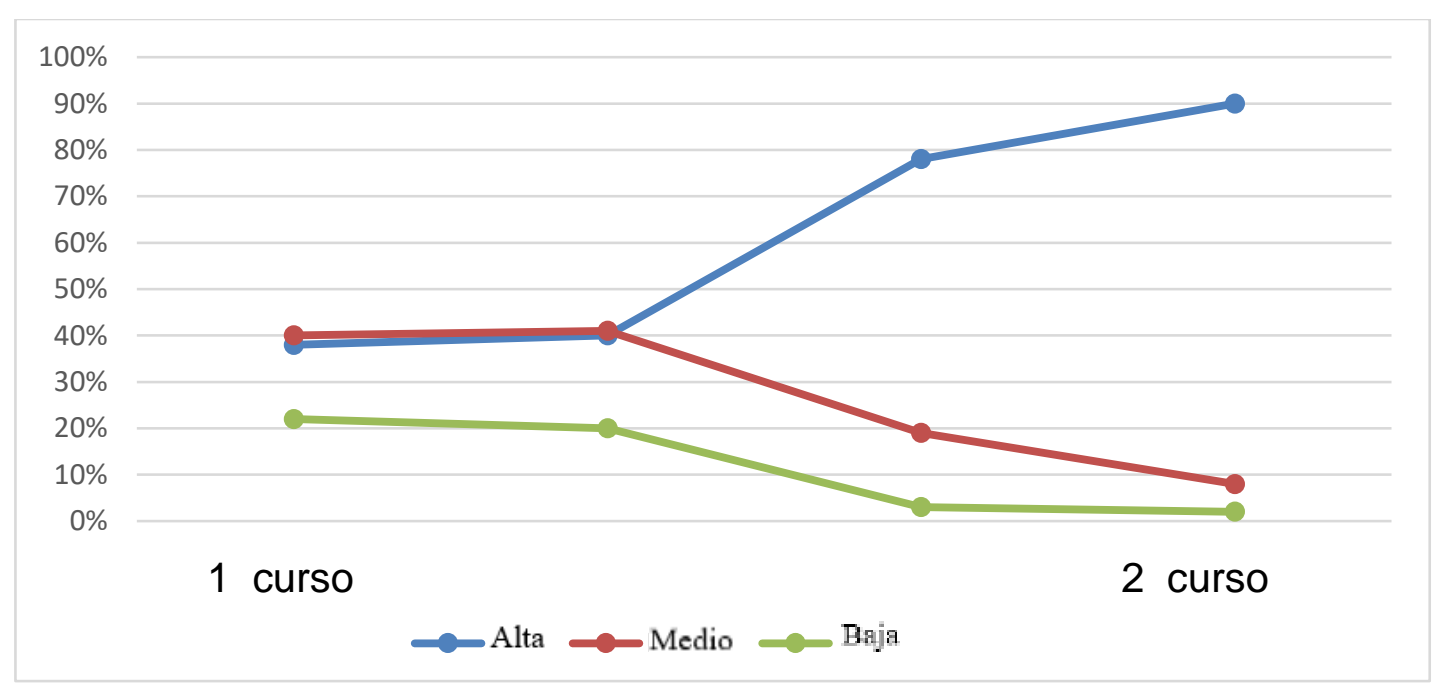

Fig. 1. Los resultados de los diagnósticos del nivel de socialización de estudiantes de instituciones de educación superior de 1 a 4 cursos.

Los resultados del diagnóstico muestran que la gran mayoría de $4^{\text {th }}$ - año los estudiantes tienen un alto nivel de socialización.

\section{Conclusiones}

El aprendizaje a distancia tiene un impacto significativo en la socialización de los estudiantes. La transición masiva al aprendizaje a distancia ha identificado una amplia gama de problemas. El estudio reveló que la socialización en los primeros años de 
formación es difícil. La imposibilidad de una interacción real alarga el proceso de adaptación de los estudiantes. Las plataformas educativas electrónicas modernas y las plataformas para la realización de seminarios web brindan una amplia gama de funciones para la capacitación, pero las nuevas capacidades técnicas, a pesar del uso activo de las tecnologías de Internet durante la escuela, plantean muchas preguntas a los estudiantes.

El estudio permitió determinar el nivel de socialización de los alumnos de $1^{\circ}$ a $4^{\circ}$ curso. Los estudiantes juniores creen que tienen las cualidades necesarias para una interacción efectiva, pero les resulta difícil adaptarse al entorno electrónico. No tienen suficiente comunicación en vivo con sus compañeros. Los cursos superiores están adaptados al proceso de aprendizaje y tienen suficiente experiencia de interacción en un entorno electrónico. Por tanto, su nivel de socialización es mucho mayor.

La socialización de los cursos junior en un entorno electrónico requiere atención adicional. En el proceso de preparación de los estudiantes de 1er año se debe involucrar a tutores, a quienes los estudiantes pueden acudir en busca de ayuda.

Los resultados obtenidos pueden ser utilizados por especialistas interesados en mejorar el proceso de adaptación de los estudiantes en la educación a distancia y la formación mixta.

\section{Referencias}

Dobudko, T.V., Korostelev, A.A., Gorbatov, S.V., Kurochkin, A.V., \& Akhmetov, L.G. (2019a). The organization of the university educational process in terms of digitalization of education. Humanities and Social Sciences Reviews, 7(4), 1148-1154.

Dobudko, T.V., Kprostelev, A.A., Pugach, O.I., Ippolitova, N. V., Khayrullina, R.G., \& Sitdikov, F.F. (2019b). Training of pedagogical education masters: Practiceoriented model. Humanities and Social Sciences Reviews, 7(4), 1155-1159.

Eliseeva, D.Yu., Fedosov, A.Yu., Agaltsova, D.V., Mnatsakanyan, O.L., Kuchmezov, Kh.Kh. (2020). The evolution of artificial intelligence and the possibility of its application in cyber games. Amazonia Investiga, 9(28), 123-129. https://amazoniainvestiga.info/index.php/amazonia/article/view/1043

Kidina, L.M. (2020). Management of the pedagogical collective in the conditions of implementing distance learning. Baltic Humanitarian Journal, 9, No 4 (33), 93-96.

Klimenko, V.A. (2012). Professional socialization of students: structural and functional model. Sociological Almanac, 3, 92-102.

Lukov, V.A., \& Lukova, E.E. (2014). On the Theory of Socialization Proposed by Franklin Giddings. Informational humanitarian portal "Knowledge. Understanding. Skill". No. 3 (May - June).

Nagovitsyn, R.S., Vaganova, O.I., Kutepov, M.M., Kosenovich, O.V, Moeseev, Yu.V., Vorotova, M.S., \& Osipov, A.Y. (2020). Interactive Technologies in Developing 
Student's Motivation in Physical Education and Sport. International Journal of Applied Exercise Physiology, 9(6), 78-85.

Nemov R.S. (1999). The basics of psychological counseling: Textbook. for stud. pedagogical universities. M.: Humanit. ed. center VLADOS, 394 p.

Nikonova, N.P., Vaganova, O.I., Smirnova, Zh.V., Bystrova, N.V., \& Markova, S.M. (2019a). Providing partnerships and promotion of additional educational services. International Journal of Applied Exercise Physiology, 7(2.1), 391-399.

Pichugina, G.A., \& Bondarchuk, A.I. (2019). Structure of the training case in the organization of the educational process. Humanitarian Balkan Research, 2(4), 5-7.

Ponachugin, A.V (2020). Practice of using interactive technologies for bachelor of applied computer science training during the COVID-19 pandemic. Vestnik of Minin University, 8(4), 6. (in Russ)

Shcerbakova, E.V., \& Shcerbakova, T.N. (2019). Experience of use of remote computer technologies at the organization of independent work of students in the conditions of a mark and rating system. Baltic Humanitarian Journal, 8, No 4 (29), 192-195.

Toktarova, V.I., \& Fedorova, S.N. (2019). Adaptation of students to learning in the conditions of the electronic information and educational environment of the university. Bulletin of the Mari State University. 13 (3(35)), 383-390.

Tyumaseva, Z.I., Orehova, I.L., \& Yakovleva, N.O. (2018). adaptational stage of the professional socialization of students of pedagogical universities. The Education and science journal, 20 (1), 75-95. (In Russ.)

Vaganova, O.I., Bakharev, N.P., Bulaeva, M.N., Lapshova, A.V., \& Kutepov, M.M. (2020a). Methods and means of realization of interaction technologies in the university. Amazonia Investiga, 9(26), 383-390. https://amazoniainvestiga.info/index.php/amazonia/article/view/1168

Vaganova, O.I., Bakharev, N.P., Kulagina, J.A., Lapshova, A.V, \& Kirillova, I.K. (2020b). Multimedia technologies in vocational education. Amazonia Investiga, 9(26), 391-398. https://amazoniainvestiga.info/index.php/amazonia/article/view/1169 